\title{
Remote consultations
}

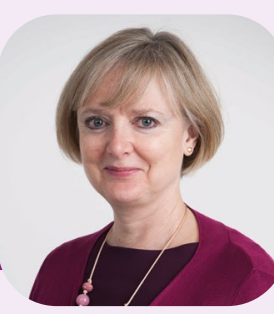

Sue $N^{\prime}$ Jie, dento-legal adviser at the DDU, discusses the dento-legal considerations of remote consultations.

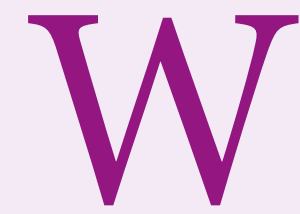

hile much in-person dentistry has resumed, the use of remote consultations has increased since the coronavirus pandemic, and continues to be part and parcel of daily practice. Remote consultations have been useful in maintaining social distancing, reducing unnecessary travel and helping to protect the most vulnerable, but it's important that remote consultations are undertaken in an ethical manner.

This is especially true if the consultation involves video conferencing or if patient images are shared to enable the patient to illustrate their dental complaint - for example, by demonstrating the site and extent of a swelling.

This article outlines the Dental Defence Union's (DDU's) advice on the many dentolegal considerations that dental professionals should keep in mind when conducting remote consultations and receiving patient information.

\section{Adhere to the GDC's guidance}

The GDC co-authored the guidance High level principles for good practice in remote consultations and prescribing ${ }^{1}$ which was developed jointly with the other healthcare regulators and this continues to apply. This guidance states that while remote consultations can 'benefit patients, save resources and help meet public demand for more convenient access to healthcare, it is also important for healthcare professionals and employers to consider the limitations of remote services.

The key point the GDC continues to make ${ }^{2}$ is to remind all dental professionals to make an appropriate risk assessment which 'should be recorded and should take into account the infection risk of COVID-19, both from and to the patient, as well the apparent seriousness of the need for treatment and the extent to which it has been possible to make a clinical assessment.
Consent and confidentiality

It's important to carry out remote consultations in a quiet, private space for both you and the patient, so that patient confidentiality is maintained.

Inform the patient if you plan to record the consultation and ensure that the patient has given consent to the recording being made. When obtaining consent, you should explain why recording the video consultation will help in providing clinical care. Respect their decision if they decline and reassure the patient, it will still be possible for the video consultation to go ahead without it being recorded.

Any recording would become part of the clinical records, so needs to be stored securely and be retrievable. Explain to the patient that the recording will not be used for any other purpose without their express permission. Document these discussions and decisions in the clinical records. It's vital to keep a record of remote consultations, in the same way as you would for a face to face consultation. While straightforward when calling or videoconferencing from the surgery, care must be taken with the security of remote access and maintaining password protections. Protocols should be in place for the secure transfer of contemporaneous records to the record system at the earliest opportunity.

Also ensure there are appropriate information security measures in place within the practice to protect personal data, such as encrypted messages and firewalls. If a patient sends additional information across to you, for example, additional photographs or a video recording, then agree with them how this information will be sent to you. If this is sent via email, then upload the information to the patient's records and delete the email and any attachments from your account.

\section{Communication}

Clear, concise and comprehensive communication is key when conducting a remote consultation. Communication can be improved by making use of digital technologies to allow an element of face to face interaction rather than a simple telephone call. When you start a video consultation, identify the patient and confirm whether you are speaking to them or their representative. Introduce yourself, explain your role and the purpose of the consultation. Look at the camera to make eye contact with the patient, rather than your screen.
Check for non-verbal cues where possible, such as hesitation or signs of distress. If this happens, slow down and check the patient is fully understanding what is being asked of them and that they are okay to continue. To avoid misunderstandings, repeat what the patient has said and ask them to confirm. If communication proves difficult and you are unable to establish a clear history of their current concerns, be prepared to convert to a face-to-face consultation where possible.

\section{Capacity}

If you feel the patient lacks capacity then you will need to seek the permission of an individual who has the legal authority to act on the patient's behalf. In the case of a child who lacks capacity to make an informed decision about a remote consultation being conducted, then you'll need the permission of someone with parental responsibility.

If the patient is an adult who lacks capacity, you must be satisfied the remote consultation is necessary, will be of benefit to them and in their best interests and seek the agreement of the individual acting on their behalf with regards to healthcare decisions.

To learn more about remote consultations during the coronavirus pandemic you can watch the DDU's remote consultations webinar, ${ }^{3}$ listen to the podcast [through the DDU app] or visit https://www.theddu.com/coronavirus.

\section{References}

1. General Dental Council. High level principles for good practice in remote consultations and prescribing. Available at: https://www. gdc-uk.org/docs/default-source/guidancedocuments/high-level-principles-remoteconsultations-and-prescribing.pdf (accessed 3 March 2021).

2. Czerniawski S. Responding to COVID-19: providing treatment in uncertain times. General Dental Council, 26 March 2020. Available at: https://www.gdc-uk.org/ news-blogs/blog/detail/blogs/2020/03/26/ responding-to-covid-19-providingtreatment-in-uncertain-times (accessed 3 March 2021).

3. Dental Defence Union. Remote consultations. 11 February 2021. Available at: https://www.theddu.com/guidanceand-advice/podcasts-and-videos/remoteconsultations (accessed 3 March 2021). 